Journal of OBJeCt TECHNOLOGY

Published by AITO - Association Internationale pour les Technologies Objets

http://www.jot.fm/

\title{
Preface to the JOT special issue containing the ECMFA 2020 selected papers
}

\author{
Richard Paige $^{\mathrm{a}}$ \\ Antonio Vallecillo ${ }^{\mathrm{b}}$ \\ a. McMaster University, Canada. \\ b. ITIS Software, Universidad de Málaga, Spain.
}

\begin{abstract}
This issue collects the papers selected to be presented at ECMFA 2020. The editors also describe the editorial and reviewing process for the conference, and reflects on how it worked.
\end{abstract}

Keywords Model-based engineering, software engineering, model transformation

\section{Introduction}

Model-Based Engineering (MBE) is an approach to the design, analysis, and development of software and systems that relies on exploiting high-level models and computer-based automation to achieve significant boosts in both productivity and quality.

The European Conference on Modelling Foundations and Applications (ECMFA) is the premier European forum dedicated to advancing the state of knowledge and fostering the application of all aspects of MBE and related approaches.

In its 16th edition, ECMFA introduced two major innovations to strengthen the scope of the community and to foster the quality of its contributions. First, the International Conference on Model Transformations (ICMT) joined forces with ECMFA, merging both into one single event that unites all aspects related to ModelBased Engineering. Second, a two-phase submission and review process was introduced, with two possible submission periods (October and February). Authors of papers not accepted in the first phase had, in most cases, the opportunity to resubmit improved versions of their work in the second submission phase. This is fully aligned with the decision made last year of publishing the ECMFA accepted papers in a platinum openaccess journal, namely, the JOT journal (http://www.jot.fm/), which is indexed by different organizations, including Scopus, DBLP, and Scientific Commons.

Although ECMFA 2020 was supposed to be held physically in Bergen, Norway, from June 22 to 26, the unfortunate pandemic caused by Covid-19 and the world-wide

Richard Paige, Antonio Vallecillo. Preface to the JOT special issue containing the ECMFA 2020 selected papers. Licensed under Attribution-NonCommercial-NoDerivatives 4.0 International (CC BY-NC-ND 4.0). In Journal of Object Technology, vol. 19, no. 2, 2020, pages e1:1-5. doi:10.5381/jot.2020.19.2.e1 
quarantine and lockdown situation prevented the conference from happening. The organizers and the Steering Committee of STAF decided to postpone the conference for one year. The next conference will be held in Bergen, in late June 2021, hosting the same or very similar set of conferences and workshops as was planned for 2020 . Nevertheless, it was also decided that the review and publication process will continue, and accepted papers will be published as planned. In addition, some kind of recognition will be made to authors of accepted papers at the 2021 event in Bergen.

This special issue contains the accepted papers at ECMFA 2020. This paper also presents some reflections on how the new double-phase review worked, the measures taken to alleviate the fact that the papers will not be presented at the conference this year, and some suggestions for future actions.

\section{The submission and review process}

\subsection{Call for Papers}

The ECMFA 2020 call for papers was issued in August 2019, allowing enough time for potential authors to prepare their manuscripts. As in previous years, we solicited papers presenting original research on all aspects of model-based engineering, distinguishing between two types of submissions:

- Foundation Papers, dealing with modeling foundations, such as metamodeling, model transformations, model engineering methods and tools, and related aspects.

- Application Papers, dealing with the application of modeling techniques, including experience reports on the use of MBE methods and tools, industrial case studies, or successful applications of MBE practices in the industry or in public administrations, with significant modeling lessons learned. All applications must have been done in real contexts and at least one of the authors of the paper must be from the company or administration where the application took place.

Artifacts are essential elements of some types of papers, since they enable the reproduction of the results, the replicability of the experiments, and contribute significantly to a better open science. In ECMFA 2020 artifacts were strongly recommended, although not strictly required. Depending on the papers, reviewers could take the existence of artifacts as a positive signal about the work. Also depending on the papers, artifacts could be required as a condition for publication.

\subsection{Review process and evaluation criteria}

The call for papers clearly stated the new review process and the paper evaluation criteria.

As traditional in ECMFA, submissions were peer-reviewed by at least three members of the Program Committee, and contributions were evaluated based on their suitability for the conference, originality, and technical soundness. They required novel and scientifically rigorous contributions to the field, supported by adequate evaluation results.

The list of topics announced in the call for papers was also made shorter and more compact, providing a more generic framework for describing the scope of the conference and allowing to focus on the main topics of interest. More precisely, the list of topics of interest included, but was not limited to: 
- Foundations of MBE and model transformations.

- Application of MBE methods, tools, and techniques to specific domains, e.g., automotive, cyber-physical systems, robotics or IoT.

- Successful use of MBE and Model Transformations in connection with other disciplines and approaches, such as Artificial Intelligence, blockchain, or Open Source.

- Educational aspects of MBE.

- Tools and initiatives for the successful adoption of MBE in industry

Contributions could be submitted to any of the two submission periods defined for the conference:

- Round 1. Submission: Oct 11, 2019; Notification: Dec 13, 2019.

- Round 2. Submission: Feb 21, 2020; Notification: April 24, 2020.

Papers submitted to the first round of review could be recommended by the Program Committee to be accepted-as-is, accepted with minor revisions, undergo major revisions (and resubmit in the second round) or rejected. Recommendations for papers submitted in the second round are for accept, minor revisions or reject.

Papers accepted at any of the two rounds with minor revisions were be given a specified period of time to perform the revisions and re-submit. The same reviewers assessed how well the revision requests had been addressed by the authors, and whether the final paper maintained or improved the level of contribution of the original submission. Revisions that significantly lessened the contribution of the work or that failed to adequately address the reviewers' original concerns resulted in the paper's rejection, as it happened with one of the papers.

Papers that were not accepted in the first round could be resubmitted to the second one, indicating how they have improved the paper to address the reviewers' criticisms. Papers not accepted in the second round but with a sufficient level of quality were be encouraged to resubmit next year, or alternatively as regular JOT articles, hence continuing their review process through the normal journal channels.

We received 11 submissions in the first round. Interestingly, the average quality of these submissions was higher than we expected. After the review process, the Program Committee decided to accept six of them with minor changes, four were asked to undergo major revisions and resubmit in the second round, and one was rejected. The five papers accepted with minor changes were given one month to prepare their revised version, i.e., until January 31, 2020. They all successfully addressed all suggested changes and improvements, and were accepted.

Another 25 papers were submitted in the second round, which occurred in the traditional ECMFA deadline of the end of February, together with the revised versions of the four papers that were asked to undergo major revisions. A revised version of the rejected paper in the first phase was also submitted, being treated as a new submission.

As a result of the second round of reviews, 12 papers were accepted with minor changes, and other two were conditionally accepted. All were given one month to address the reviewers' comments and suggestions. At the end, 11 papers were accepted in this phase, since one of the conditionally accepted papers was finally rejected. 
In summary, the Program Committee of ECMFA 2020 accepted 19 papers, which are the ones that comprise this special issue. Given that there will be no physical presentations of the papers at the conference, we asked authors of accepted papers to produce a brief teaser video of their work, in order to summarize the paper contributions and its major highlights. These companion videos are published together with the papers in the JOT journal.

\subsection{Some reflections and lessons learned}

The two stage review process worked quite well for ECMFA 2020. Authors of some phase-one papers received earlier notification than they normally would. Authors of other phase-one papers received detailed feedback - of the quality of a typical journal review process - and had the opportunity to revise and resubmit as they would with any journal. Also, a good number of papers was submitted for the Phase 2 deadline in February (though such papers followed a traditional conference review model, leading to decisions of accept or reject). The relationship with JOT allowed papers to be accepted and typeset quickly and efficiently, avoiding the typical long period between notification and publication seen in many conferences. The only slight complication that the chairs experienced was in making Easychair fit with the two-phase process. Overall, the chairs were very happy with the two phase approach, believe that it led to higher quality reviews, a more engaged program committee and author community, and ultimately a higher acceptance rate. We strongly believe that this model - which aims to make conferences more like journals, in terms of publication process - is likely to be the future of publication in software engineering.

\subsection{Next steps}

As the authors of ECMFA 2020 papers did not have an opportunity to present their work in person, we are exploring alternatives for recognizing authors at the next ECMFA conference in Bergen in 2021. While the current chairs and the STAF steering committee have some ideas on how to do this, they would welcome suggestions from the community (please email the chairs with any ideas).

\subsection{Acknowledgements}

We would like to thank the Organization of the ECMFA and STAF conferences for their continuous help and support, which have greatly simplified our tasks as Program Committee Chairs by providing us with all the supporting review tools, Web infrastructure, and excellent publicity.

We are sincerely thankful to authors of all submitted papers for trusting ECMFA as a publication venue for their work. We hope that the feedback and suggestions provided by the Program Committee members have helped them improve their research, no matter if they were finally accepted or not.

Our gratitude also goes to all the Program Committee members and external reviewers for the timely manner in which they assisted in choosing and making suggestions to improve the selected papers, and for their helpful and insightful comments. We are aware of all the time and effort involved in producing such constructive reports, and in helping authors improve their papers.

Finally, we show our appreciation to the JOT editorial office and in particular to Alfonso Pierantonio, for their excellent support in the preparation of this special issue. 


\section{About the authors}

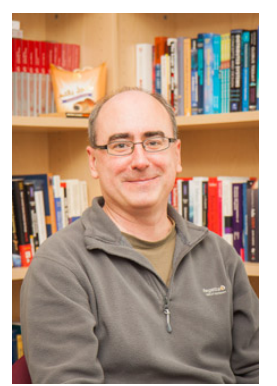

Richard Paige is a full Professor at McMaster University, where he is director of the Centre for Software Certification. His research interests are in Model-Based Systems Engineering, certification, safety, data analytics, and formal methods. Contact him at paigeri@mcmaster.ca or visit https://www.eng.mcmaster.ca/ cas/people/faculty/richard-paige

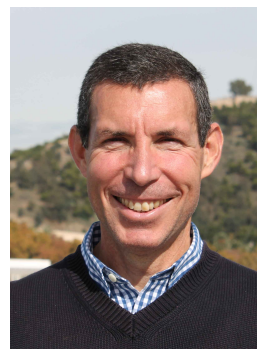

Antonio Vallecillo is full Professor at the University of Málaga, where he leads the Atenea Research Group on Software and Systems Modeling. His research interests include Open Distributed Processing, Model-based Engineering and Software Quality. Contact him at av@lcc.uma.es, or visit https://lcc.uma.es/ av. 